\title{
Prediction of Incipient Faults in Underground Power Cables Utilizing S-Transform and Support Vector Regression
}

\author{
M. F. Faisal ${ }^{1}$, A. Mohamed ${ }^{1}$, H. Shareef ${ }^{1}$ \\ ${ }^{1}$ Department of Electrical, Electronic and Systems Engineering \\ Universiti Kebangsaan Malaysia, Bangi, Malaysia \\ azah@eng.ukm.my
}

\begin{abstract}
Incipient faults usually emerge from partial discharges which eventually cause insulation degradation between two insulated cable cores. Early detection of incipient faults is of particular importance because insulation defects caused by incipient faults may lead to permanent faults in underground distribution networks. This paper presents a novel approach using S-transform and support vector regression to predict the occurrence of incipient faults in underground cable networks. The results of this study proved that the new approach is capable to predict the existence of partial discharges in underground power cable systems in Malaysia.
\end{abstract}

Keywords: Incipient fault, Partial discharge, S-transform, Support Vector Regression

\section{Introduction}

Short circuits may occur due to electrical stress or contamination that progressively weakens the integrity of underground cables and over time will lead to insulation failure. Examples of insulation damage are abrasions caused by digging or ground seizing and corrosion damage from salt or oil exposure. Most often the insulation undergoes a gradual aging process before a permanent short circuit fault happens. This type of short circuits is usually referred as incipient faults because the electrical property of the insulation alters adversely and incipient-like behavior commences. Incipient faults may be defined as nonperiodic, asymmetric and sporadic arcing currents, which are random in magnitude and could involve sporadic bursts. Such faults are self-clearing arcing faults which does not draw high current during initial stages but occur very frequently prior to a permanent failure.

The common causes of the electrical stress are due to partial discharge (PD) activities. A $\mathrm{PD}$ is a localized dielectric breakdown of a small portion of a solid or liquid electrical insulation system under high voltage stress. PD partially bridges the insulation between conductors and can occur adjacent to a conductor. PD is often detected as small electrical sparks that occur within the electric insulation of switchgear, cables and transformers and is limited in extent, so it does not immediately cause full insulation breakdown. PD is often initiated within gas voids enclosed in solid insulation, or in bubbles within a liquid insulating material, such as voids in an epoxy insulator, or gas bubbles dissolved within transformer oil [1]. As the gas within the void has a dielectric constant much less than the surrounding material, it will experience a significantly higher electric field. When this becomes high enough to cause electrical breakdown in the gas, a PD occurs.

Partial discharges at the interface of two media are commonly called surface discharges and produce the well-known surface tracking [2]. An example of the signatures of surface tracking on a cable termination is shown in Figure 1. Surface tracking at the interface between solid insulation and air frequently result from the following process. At each end of the potential gradient, any irregular or sharp needle-like point will have a stronger electric field. This stronger electric field ionizes the surrounding air, therefore causing this air to be conductive

Received: December $9^{\text {th }}, 2011$. Accepted: June $27^{\text {th }}, 2012$ 
and partial discharges may exist in this region. At some point, the addition of contamination and moisture may create a leakage path to ground. In paper-insulated medium-voltage cables, the repetitive PD will eventually cause permanent chemical changes within the affected paper layers and impregnating dielectric fluid. Over time, partially conducting carbonized electrical trees are formed. This places greater stress on the remaining insulation, leading to further growth of the damaged region, resistive heating along the tree, and further charring [3]. This eventually culminates in a complete dielectric failure of the cable and, typically causes an electrical explosion. An example on the effect of electrical tree on a cable termination is shown in Figure 2.

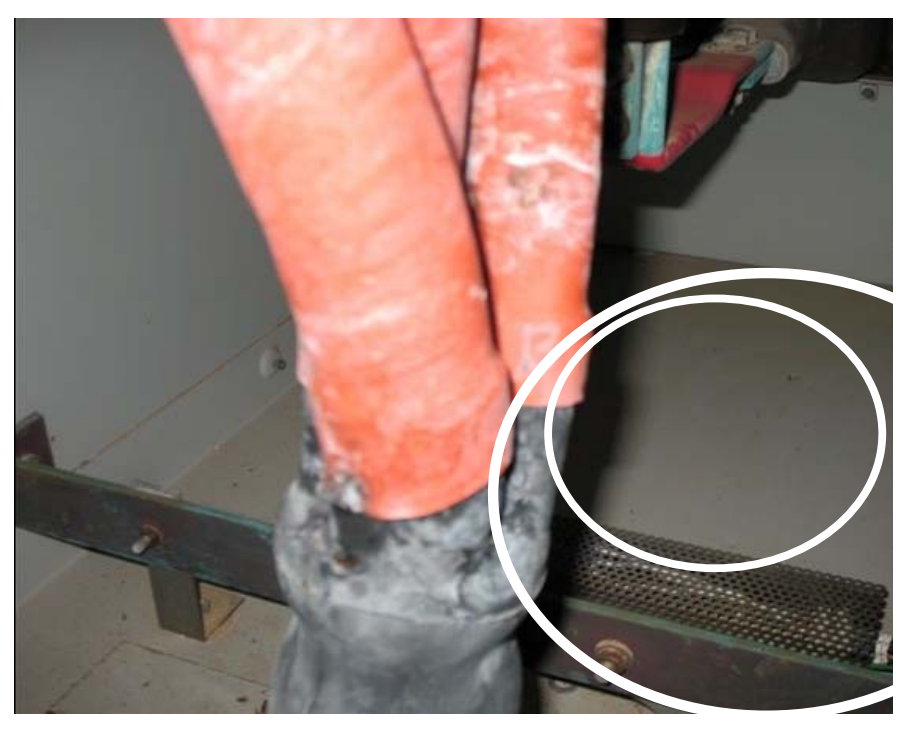

Figure 1. Example of surface tracking at a cable termination

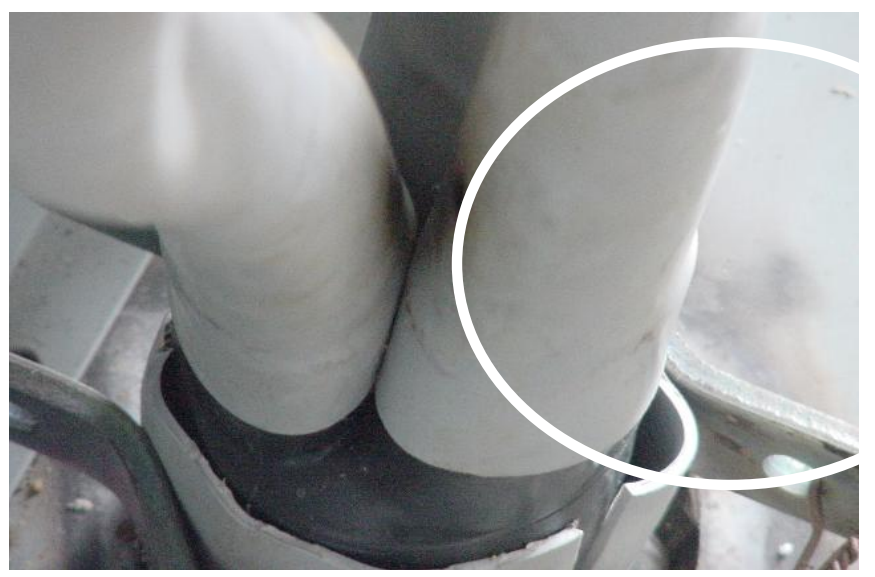

Figure 2. Example of signature of insulation failure due to electrical tree

A significant increase either in the PD level or in the developing rate of PD activity can provide an early indication of an incipient fault condition. Operational experience, suggests that it is important to isolate the underground power cable suspected of incipient fault after detecting the first symptom of a PD. Isolating the underground cable network can limit the 
overall energy at the point of fault and also reduce the occurrence of repeated voltage transients in the system. The detection and isolation of incipient faults in a power supply system is essential for ensuring safe, reliable, and efficient operation of consumers' installations. Incipient faults are predictable and avoidable if the degradation processes are known and detectable.

One of the earliest known and practical techniques for detecting incipient fault in underground cable system was developed by Weeks and Steiner [4]. The technique detected the existence of electromagnetic noises that appears at both ends of power cable terminals to indicate the existence of incipient fault. The noise generated at the sites of deterioration will propagate along the cable in both directions from its origin. If the noise signal recorded at one end of the cables correlate with the other noise signals recorded at the other end, then incipient fault is detected. In [5], it was observed that there were major differences between PD and electromagnetic noises as detected by capacitive couplers installed at the phase terminals. The pulse response is greatest in the phase which has a PD whereas for noise, the initial signal magnitude is much the same on all three phases. Therefore, monitoring electromagnetic noise for detecting incipient fault may not yield accurate results as monitoring for PD activity.

Kim et. al. in [6] applied statistical approach to identify symptoms related to incipient faults in distribution networks, using the trend analysis method called the Laplace trend test. The existence of incipient fault is detected if the Laplace test index is greater than 1.96. The drawback of this technique is that the detection of incipient fault can only be determined by accessing both the fault and event data. Another method for detecting PD using electrical signals emitted from an underground cable was introduced by Dorris et. al [7]. Here, the detected small and fast current pulses are associated with water treeing in underground cable systems. A water tree is a chemical degradation of polymeric insulation such as XLPE that only occurs in the presence of water and electrical stress. The study showed that water treeing can induce certain current pulses that can be detected as image currents induced in the adjacent ground electrodes. The disadvantage of this technique is related to electrical noises that could exceed the set threshold and create a record resembling fast current pulses, thus, giving a misleading result in the detection of PD. Another similar technique that involved measuring the current pulses during pre-PD conditions was developed in [3]. The measurement of these nondestructive current pulses has practical significance, in which it can detect true PD occurrence. The non-destructive current pulses were also related to the existence of space charge in underground cable systems which were formed under de stress in dielectrics during local dielectric breakdown or electrical tree initiation [8]. It was noted that there is a link between space charge and electrical tree in which the onset of electrical treeing is represented by current pulses which detect incipient fault in underground cable systems.

Ahmad and Srinivas in [9] used a digital spectrum analyzer to detect on-line PD in which the analyzer was equipped with a high frequency preamplifier and high frequency inductive sensors installed in underground cable systems. The existence of PD was identified based on the detection of high frequency electromagnetic pulses in both the voltage and current signals in underground cable. The detection of high frequency electromagnetic pulses would indicate the existence of incipient fault. However, special care must be given to distinguish between external electromagnetic noise and electromagnetic signals produced by the PD activities inside the cable. On-line PD testing called as the CableWise Technology uses a digital spectrum analyzer for detecting high frequency signals both prior to and during PD inception [10]. From the signal information, the CableWise technique will rank the sections of the underground cable according to the degree of degradation with respect to the VHF values. The significance of the high frequency electromagnetic pulses in the detection of PD was also highlighted in [11].

New approaches have been developed to detect incipient fault based on diagnosis of current signals recorded from underground power cables [12]. Underground cables are known to exhibit incipient, self-clearing arcing faults prior to failing permanently in which the incipient fault typically lasts between half-cycle $(10 \mathrm{~ms})$ to 3 half-cycles $(30 \mathrm{~ms})$ in a $50 \mathrm{~Hz}$ system. 
During such period of PD, high frequency components are present in the current waveforms. Eventually when the insulating layer is broken, a high fault current of fundamental frequency is created. During the occurrence of the incipient faults, the values of neutral current and the fault component in the affected phase will correlate thus confirming the detection of incipient faults. The accuracy of this method is very much dependent on the sensitivity of the available relays and current transformers. Butler in [13] applied a similar methodology, which includes an expert system for detection of incipient fault and predictive maintenance. Abnormalities due to incipient faults are recognized through comparisons with the existing knowledge data base. However, the success of this method is very much depends on the knowledge database.

Morghe et. al in [14] applied time and frequency domain analysis on actual scenarios of a certain class of incipient faults leading to an eventual splice failure in an underground distribution system. The time domain was analyzed by converting the current waveforms into root mean square (rms) values. From the rms, the value and duration of the transient current was evaluated to justify the existence of incipient fault. The frequency domain analysis was performed by applying the Fast Fourier Transform (FFT) on the incipient current cycle to provide a view of the harmonics present in the faulted cycle.

In a real practical situation, the harsh noise environment in the electrical system is one of the limiting factors in the detection of PD and electrical tree. Therefore, in order to obtain accurate detection of incipient fault, it is recommended that power quality recorders are to be used to record all abnormal conducted signals in the power systems. Power quality recorders with high sampling rate can detect and record minor abnormalities in the current and voltage waveforms. Signal processing techniques must then be used in processing the recorded signals. The selection of signal processing technique is very important as the waveforms recorded during the occurrence of PD are non stationary and noise polluted. The application of FFT for the detection of disturbances will be inaccurate as the FFT only gives the frequency components that exist in the signal [15]. Another relatively new signal processing technique known as the wavelet transform which gives time localization of the spectral components of signals has been applied for analyzing power quality disturbance signals [16]. However, it is reported that the performance of the wavelet transform is less superior compared to the new time frequency technique known as the S-transform (ST) [17]. In this paper, a novel approach to predict the existence of incipient fault in the existing underground cable system in Malaysia is proposed using the ST and the support vector regression (SVR) technique. The excellent time-frequency resolution characteristic of the ST makes it an attractive candidate for the analysis of power quality (PQ) disturbances under noisy condition [18]. The SVR is considered as a relatively new supervised learning technique for predicting the existence of incipient faults.

\section{Proposed Method for Predicting Incipient Fault}

The novel approach for predicting incipient fault in a distribution system is described in terms of a block diagram as shown in Figure 3. Initially, the voltage disturbances are assumed to be recorded via the on-line power quality monitoring system (PQMS) shown in Figure 4. As shown in Figure 4, the online PQMS which comprises of a power quality recorder (PQR), is installed in a substation to capture both the voltage and current events at the $33 \mathrm{kV}$ busbar originating from the distribution feeders (DF1 to DF4) and the transmission feeders (TF1 and TF2). The data recorded will be sent to the PQMS server via a fibreoptic communication system. The PQMS runs continuously and monitors the bus and feeder for abnormalities predefined and programmed into the triggering logic. The PQMS will also send the summary of the disturbance in text format via short messaging system to the power utility engineers.

Next, the ST will process the voltage disturbance data to extract the useful features of the disturbance. These features will then be applied to the SVR for predicting the incipient faults in a distribution system. The results of SVR are classified for predicting incipient faults and classes of power quality disturbances. 


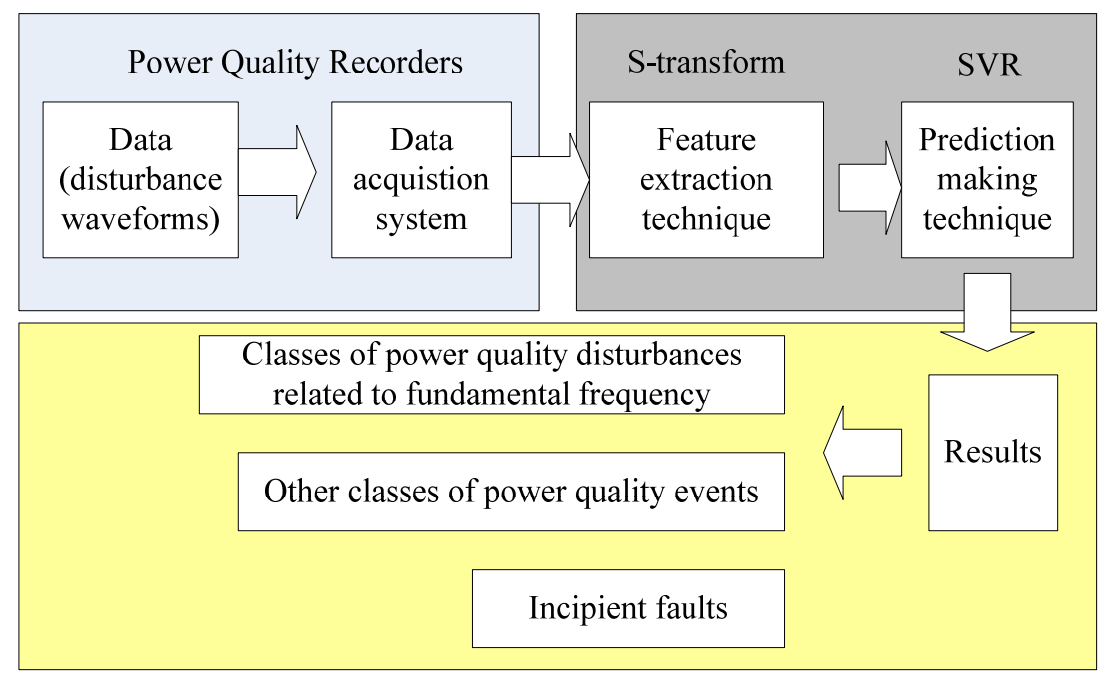

Figure 3. Process flow for the prediction of incipient faults

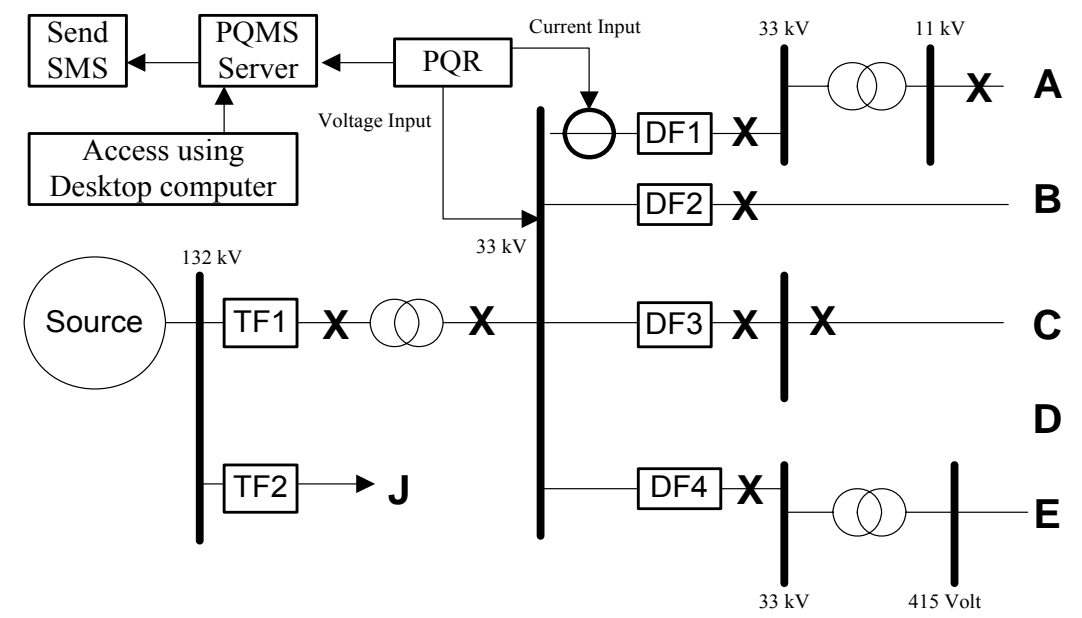

Figure 4. On-line power quality monitoring system (PQMS)

\section{S-Transform for Detecting Incipient Faults}

The S-transform is considered as one of the most recent signal processing techniques that produces a time-frequency representation of a time series signal [19]. It is similar to the wavelet transform (WT) by having progressive resolution but unlike the WT, the ST retains absolutely referenced phase information and has a frequency invariant amplitude response. The ST is an extension of the continuous WT (CWT) in which it is based on a moving and scalable localizing Gaussian window. The ST for a function, $h(t)$, can be defined as a CWT for function $h(t)$ multiplied by a phase correction factor, $e^{-i 2 \pi f \tau}$ [20]. To derive the ST from the 
CWT, firstly consider the CWT which is a series of correlations of the time series with a function called as wavelet and it is given as:

$$
W(\tau, d)=\int_{-\infty}^{\infty} h(t) \omega(t-\tau, d) d t
$$

where $\omega(t-\tau, d)$ is the mother wavelet, $t$ and $\tau$ are both time, and the dilation factor $d$ is the inverse of the frequency, $f$.

The mother wavelet can be further expressed as:-

$$
\omega(t, f)=\frac{|f|}{\sqrt{2 \pi}} e^{-\frac{t^{2} f^{2}}{2}} e^{-i 2 \pi f t}
$$

According to (2), the mother wavelet does not satisfy the admissibility condition of having a zero mean, and therefore it is not strictly a CWT [21]. The ST is obtained by multiplying the CWT, $W(\tau, d)$ with a phase factor. Substituting (2) into (1), we get the ST equation which is given as:

$$
S(\tau, f)=\frac{|f|}{\sqrt{2 \pi}} \int_{-\infty}^{\infty} h(t) e^{-\frac{(t-\tau)^{2}}{2}} e^{-i 2 \pi f t} d t
$$

The phase factor in (3) is a phase correction of the CWT. It eliminates the concept of wavelet analysis by separating the mother wavelet into two parts, which are, the slowly varying envelope or the Gaussian function which localizes in time, and the oscillatory exponential kernel, $e^{-i 2 \pi f t}$ which selects the frequency being, localized [20]. The ST can be interpreted as phase-corrected CWT and it generates contours, which are suitable for detection by simple visual inspection unlike the CWT transform that requires specific methods like standard multiresolution analysis [22].

The ST distinguishes itself from the many time-frequency representations available by uniquely combining progressive resolution with absolutely referenced phase information. It is known that progressive resolution gives a fundamentally sounder time-frequency representation. The ST has unique properties in which it uniquely combines frequency dependent resolution with absolutely reference phase, so that the time average of the ST equals the Fourier spectrum. It simultaneously estimates the local amplitude spectrum and the local phase spectrum, whereas the CWT approach is only capable of probing the local amplitude and power spectrum. It independently probes the positive frequency spectrum and the negative frequency spectrum, whereas many wavelet approaches are incapable of being applied to a complex time series. The output of the ST is an M x N matrix named as the S-matrix whose rows $(\mathrm{M})$ pertain to frequency and columns $(\mathrm{N})$ to time. Each element of the S-matrix is a complex number. The information in the S-matrix can be plotted as time-frequency contours. The advantages and disadvantages of Wavelet and $\mathrm{S}$ transform are summarized in Table 1.

As mentioned earlier, incipient faults are self-clearing arcing faults which will occur very frequently prior to failing permanently. Incipient faults can also be categorized as nonpermanent fault in which these events typically last for one half-cycle and extinguish at the first natural zero crossing of the current. The magnitude of the half-cycle event is primarily dependent on the location of the fault on the distribution network. The common causes of incipient faults are PD activities in the cable compartment. Normally, when PD is initiated, high frequency transient current pulses will appear and persist for nano-seconds to milliseconds 
then disappear and reappear repeatedly [4]. The excellent time-frequency resolution of the ST makes it an attractive candidate for detecting incipient faults.

Table 1. Comparison of Wavelet and S-Transform

\begin{tabular}{|c|c|c|}
\hline & Wavelet Transform & S-Transform \\
\hline \multirow{3}{*}{ Advantages } & $\begin{array}{l}\text { Every spectral component is not resolved } \\
\text { equally as was the case in the short time } \\
\text { Fourier transform }\end{array}$ & $\begin{array}{l}\text { Phase correction factor is in cooperated to } \\
\text { enhance the CWT }\end{array}$ \\
\hline & $\begin{array}{l}\text { Does not require to take Fourier transforms of } \\
\text { the windowed signals }\end{array}$ & More suitable to noisy non stationary signals \\
\hline & $\begin{array}{l}\text { The width of the window is changed as the } \\
\text { transform is computed for every single spectral } \\
\text { component. Therefore it is suitable to non } \\
\text { stationary signals }\end{array}$ & $\begin{array}{l}\text { It has a frequency dependent resolution that } \\
\text { simultaneously localizes the magnitude and } \\
\text { phase spectra }\end{array}$ \\
\hline \multirow{4}{*}{ Disadvantages } & $\begin{array}{l}\text { Need to isolate the noise in the signal to obtain } \\
\text { appropriate frequency resolution }\end{array}$ & $\begin{array}{l}\text { Computation may take anywhere from a } \\
\text { couple seconds to couple hours depending } \\
\text { on your signal size and the resolution }\end{array}$ \\
\hline & $\begin{array}{l}\text { Many window functions (wavelets)are } \\
\text { required }\end{array}$ & $\begin{array}{l}\text { Computer may take a lot of memory space } \\
\text { depending on your signal size and the } \\
\text { resolution }\end{array}$ \\
\hline & $\begin{array}{l}\text { Computation may take anywhere from a } \\
\text { couple seconds to couple hours depending on } \\
\text { your signal size and the resolution }\end{array}$ & $\begin{array}{l}\text { Better understanding of the time-local } \\
\text { properties of the time series is required }\end{array}$ \\
\hline & $\begin{array}{l}\text { Phase information of the signal is not } \\
\text { considered explicitly }\end{array}$ & - \\
\hline
\end{tabular}

\section{A. S-transform for Feature Extraction of Incipient Faults}

Feature extraction is a preprocessing operation that transforms a pattern from its original form to a new form suitable for further processing. A set of features that are commonly selected to analyze a set of data are based on standard mathematical statistical indices i.e. the maximum, minimum, standard deviation and mean values [18]. In this study, the first step in performing feature extraction is by mapping the voltage and current signals into its ST domain. Features that characterize incipient faults of a disturbance signal are extracted from the ST analysis in terms of time-frequency representation (TFR) curve which is in the form of an ST contour. A TFR curve or ST contour represents the energy distribution at different frequency bands over a certain period of time. The magnitude of the ST contours can be extracted from the S-matrix by isolating the maximum values of the elements present in the S-matrix. Hence, two new indices based on the ST are developed for performing feature extraction. The first index is named the ST magnitude-time voltage (STMV) and the second index is named the ST frequency-time voltage (STFV). The derivation of the STMV and STFV indices for one of the phases (red phase) is explained below:-

First, the S-transformed version of red phase voltage can be obtained as:

$$
\overline{S_{R} V_{i, j}}=\frac{|f|}{\sqrt{2 \pi}} \int_{-\infty}^{\infty} v_{R}(t) e^{-\frac{(t-\tau)^{2}}{2}} e^{-i 2 \pi f t} d t
$$

where

$$
\begin{aligned}
& \overline{S_{R} V_{i, j}}: \text { S-matrix for voltage } V_{R} \\
& \mathrm{i}=1 \ldots \mathrm{N}: \text { number of columns } \\
& \mathrm{j}=1 \ldots \mathrm{M}: \text { number of rows }
\end{aligned}
$$


Then, the STMV for the red phase which is the maximum value appearing in all the columns of the S-matrix, can be obtained as:

$$
\overline{V_{S T M V R}}=\max \left(\overline{S_{R} V_{i}}\right)
$$

where $\overline{V_{S T M V R}}$ is the vector for the maximum value for all the columns in the S-matrix for the red phase voltage. The STMV plot indicates the changes in amplitude resolution. The STMV can be used to characterize incipient faults of a disturbance signal in terms of time-amplitude representation.

Similarly, considering the vectors of the maximum value for all the rows in the S-matrix for the voltage of red phase, the STFV for the red, is derived as follows:

$$
\overline{V_{S T F V R}}=\max \left(\overline{\left.S_{R} V_{j}\right)}\right.
$$

where $\overline{V_{S T F V R}}$ is the vector for the maximum value for all the rows in the S-matrix for the red phase voltage. The STFV plot indicates the changes in frequency resolution. The STFV can be used to characterize incipient faults of a disturbance signal in terms of time-frequency representation. One can derive STMV and STFV for yellow and blue phases can be obtained by following a similar procedure.
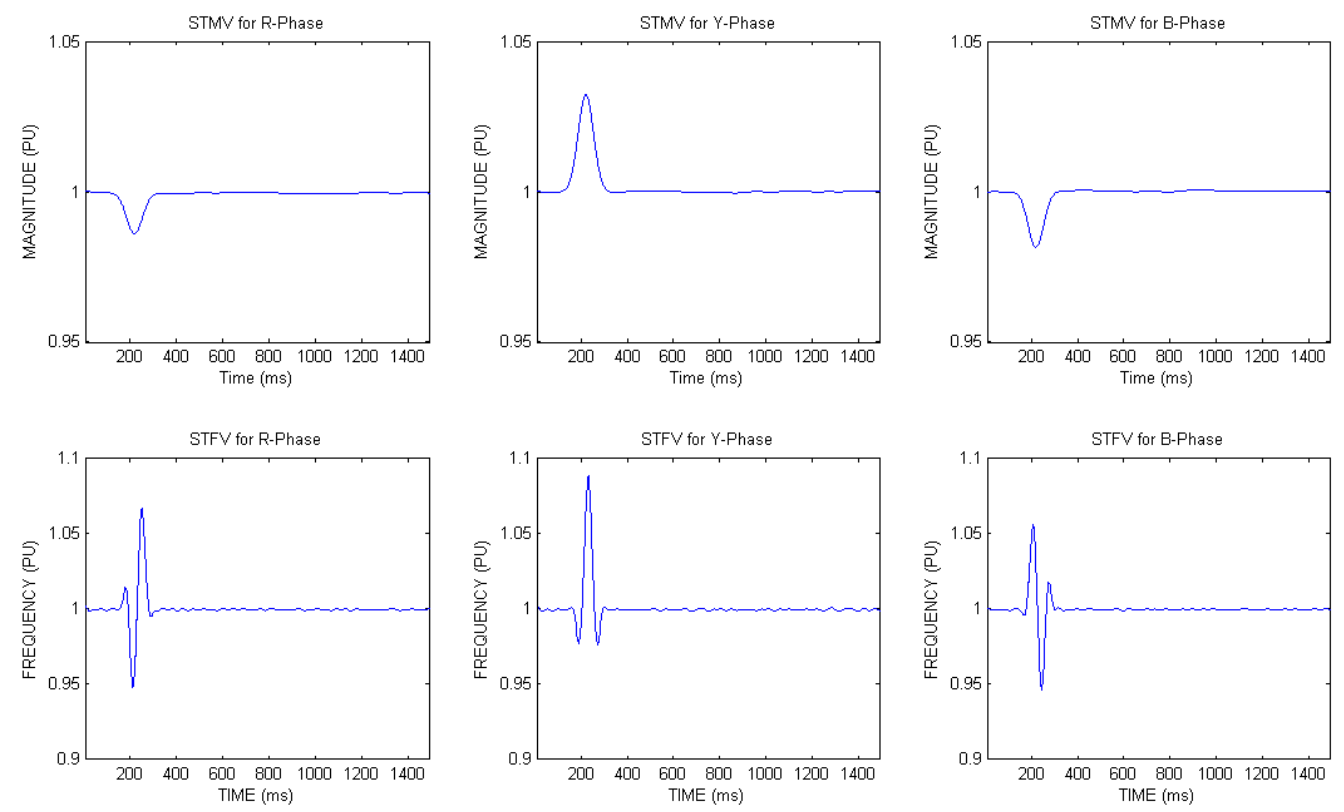

Figure 5. The STMV and STFV plots for an incipient fault for Example signal 1

In Figure 5, both the STMV and STFV plots indicates a very short duration increase in the yellow phase voltage and frequency values which signified the existence of high frequency current caused by an incipient fault in the cable termination since similar voltage spikes was observed randomly throughout monitoring period. The minimum STMV values for the red and 
blue phases are greater than 0.95 per unit which indicated no occurrences of voltage sags. The maximum value of the STMV for the yellow phase is less than 1.17 per unit and therefore, no voltage swell exists in the phase voltage. The maximum values of the STMV and STFV are 1.032 and 1.09 per unit, respectively.

Another example for STMV and STFV plots is shown in Figure 6. The minimum STMV values for the yellow and blue phases are greater than 0.95 per unit which indicated no occurrences of voltage sags. The maximum value of the STMV for the yellow phase is less than 1.17 per unit and therefore, no voltage swell exists in the phase voltage. In this example, the maximum values of the STMV and STFV are 1.032 and 1.15 per unit, respectively. The common ranges for the STMV and STFV for detecting incipient faults are shown in Table 2.

Table 2. STMV and STFV values for incipient faults

\begin{tabular}{ccc}
\hline Number of data & $\begin{array}{c}\text { Ranges of STMV to detect } \\
\text { incipient fault }\end{array}$ & $\begin{array}{c}\text { Ranges of STFV to detect } \\
\text { incipient fault }\end{array}$ \\
\hline 100 & $1.032<$ STMV $<1.17$ & $1.08<$ STFV $<1.17$ \\
\hline
\end{tabular}

To further categorize the types of faults, two new features, F1 and F2, are derived from the STFV. Feature F1 or STDV $V_{X}$ values are the sum of the standard deviations for the STFV plots for all the phase voltages. The feature, F1 is expressed as follows:

$$
F 1=\sqrt{\left[S T D V_{R}^{2}+S T D V_{Y}^{2}+S T D V_{B}^{2}\right]}
$$

where $S T D V_{R}$ is the standard deviation of the STFV plots for red phase voltage waveform, $S T D V_{Y}$ is the standard deviation of the STFV plots for yellow phase voltage waveform, and $S T D V_{B}$ is the standard deviation of the STFV plots for blue phase voltage waveform.
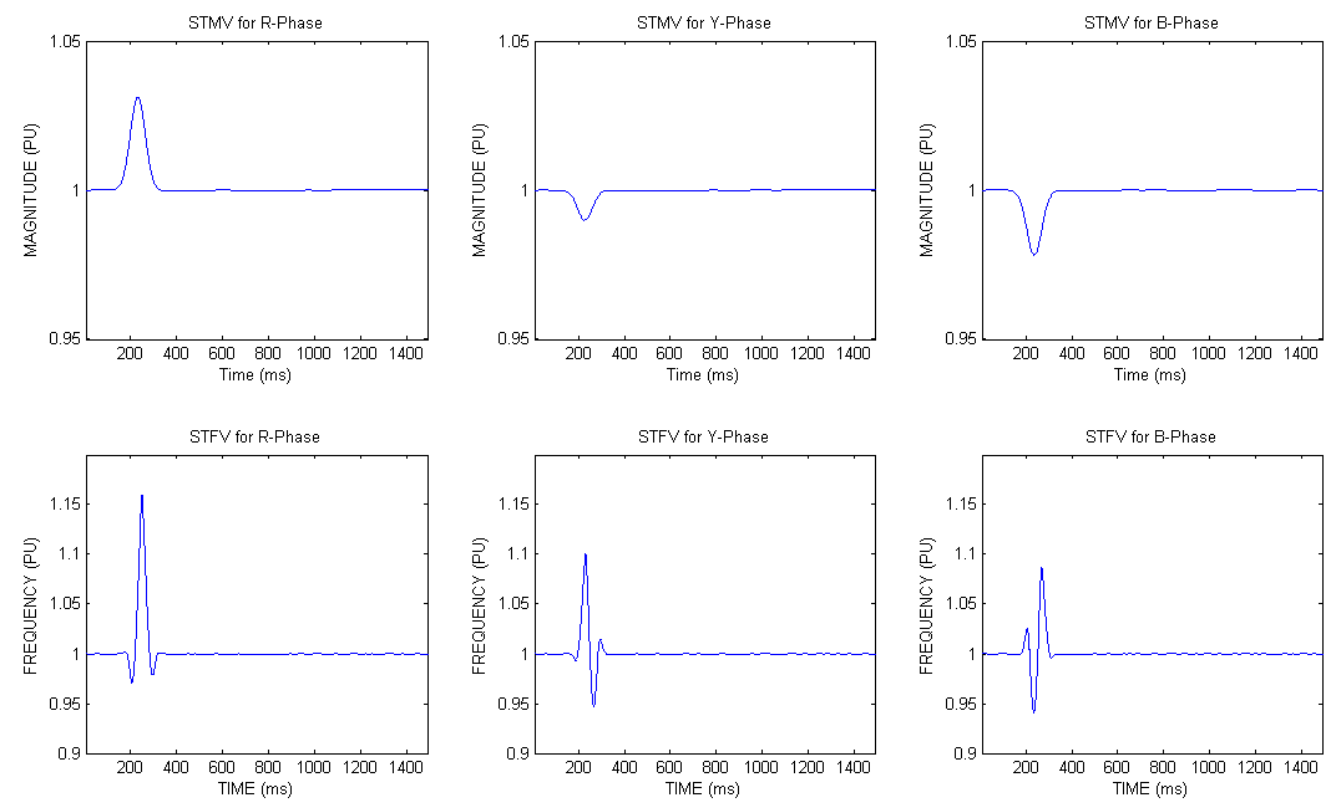

Figure 6. The STMV and STFV plots for an incipient fault for Example signal 2 The next feature, F2, is the difference between the maximum and minimum values of the STFV plots which is given by, 


$$
F 2=\sqrt{\left[M A X V_{R}^{2}+M A X V_{Y}^{2}+M A X V_{B}^{2}\right]}
$$

where

$$
\begin{aligned}
& M A X V_{R}=\max \left(S T F V_{R}\right)-\min \left(S T F V_{R}\right) \\
& M A X V_{Y}=\max \left(S T F V_{Y}\right)-\min \left(S T F V_{Y}\right) \\
& M A X V_{B}=\max \left(S T F V_{B}\right)-\min \left(S T F V_{B}\right)
\end{aligned}
$$

Features, F1 and F2 are applied in the prediction of incipient faults. These features were tested on 105 sets of power quality disturbance data comprising of both voltage sags and voltage swells. The respective values of the features F1 and F2 are then calculated and plotted on a scatter plot. From the scatter plots, the values of F1 for classifying incipient fault is such that it has to be less than 0.027 and for classifying non-incipient fault it has to be greater than 0.027 . The values of F2 for classifying incipient fault should be less than 0.30 and for classifying non-incipient fault it has to be greater than 0.30 . Table 3 shows the values of features, F1 and F2 for classifying incipient and non-incipient faults.

Table 3. Feature F1 for classifying incipient and non-incipient faults

\begin{tabular}{clcc}
\hline Feature & \multicolumn{1}{c}{ Description } & Non-incipient fault & \multicolumn{1}{c}{$\begin{array}{c}\text { Incipient } \\
\text { fault }\end{array}$} \\
\hline F1 & $\begin{array}{l}\text { Square root of the sum of } \\
\text { the standard deviations for }\end{array}$ & $0.0270<\mathrm{F} 1<0.100$ & $0.00<\mathrm{F} 1<0.0270$ \\
F2 & $\begin{array}{l}\text { STFV } \\
\text { Square root of the } \\
\text { difference between the } \\
\text { maximum and minimum } \\
\text { values of the STFV }\end{array}$ & $0.300<\mathrm{F} 2<0.400$ & $0.00<\mathrm{F} 2<0.300$ \\
\hline
\end{tabular}

\section{Application of Support Vector Regression for Prediction of Incipient Faults}

Initially, SVMs were developed to solve classification problems, but recently, they have been extended to the regression problem domain. The term SVM is typically used to describe classification with support vector methods, while support vector regression (SVR) is used to describe regression with support vector methods [23].

By viewing input data as two sets of vectors in an n-dimensional space, an SVM will construct a separating hyperplane in that space, one that maximizes the margin between two data sets. To calculate the margin, two parallel hyperplanes are constructed, one on each side of a separating hyperplane, which are "pushed up against" the two data sets. Intuitively, a good separation is achieved by the hyperplane that has the largest distance to the neighboring data points of both classes, since, in general, the larger the margin is, the better the generalization error of the classifier. Consider a sample training data which are a set of points of the form:

$$
D=\left\{\left(x_{i}, c_{i}\right) \mid x_{i} \in R^{p}, c_{i} \in\{-1,1\}_{i=1}^{n}\right.
$$

where $c_{i}$ is either 1 or -1 , indicating the class to which the point $X_{i}$ belongs. Each $X_{i}$ is a $p$-dimensional real vector. 
To classify the data set, a maximum-margin hyperplane is required that can divide the points having $c_{i}=1$ from those having $c_{i}=-1$. The classification is done by means of a dividing hyperplane, which takes the form of the equation:

$$
f(x)=w^{T} x-b
$$

where $w$ is orthogonal weight vector $\left[w_{1}, w_{2}, \ldots . w_{n}\right]^{T}$ and $b$ is a scalar used to increase the margin. The vector $w$ is a normal vector and it is perpendicular to the hyperplane. The parameter $b$ determines the offset of the hyperplane from the origin along the normal vector $w$. The dividing hyperplane is achieved when $w \cdot X-b=0$. Without this parameter, the hyperplane created will be restricted to pass through the origin only. Thus, the position of the dividing hyperplane is determined by the vector $\mathrm{w}$ and scalar $\mathrm{b}$. In order to distinguish between the two classes, label $y_{i}$ is used as:

$$
\begin{array}{ll}
y_{i}=+1 & \text { if } x \text { belong to Class } 1 \\
y_{i}=-1 & \text { if } x \text { belong to Class } 2
\end{array}
$$

In other words, the dividing hyperplane has to follow the following constraints:

$$
\begin{array}{lll}
f\left(x_{i}\right) \geq 0, & \text { if } & y_{i}=+1 \\
f\left(x_{i}\right) \leq 0, & \text { if } & y_{i}=-1
\end{array}
$$

Thus, two parallel hyperplanes are created on each side of the dividing hyperplane, satisfying the above constraints. The hyperplanes can be described by the following equations:

$$
w \cdot x-b=+1 \text { and } w \cdot x-b=-1
$$

The samples on the margin in Figure 7 are called the support vectors. By using geometry, the distance between these two hyperplanes is $\frac{2}{|w|}$. The optimal dividing hyperplane is obtained by maximizing the margin $\frac{2}{|w|}$, such that there are no points between the above parallel hyperplanes and thus minimizing $|w|[24,25]$.

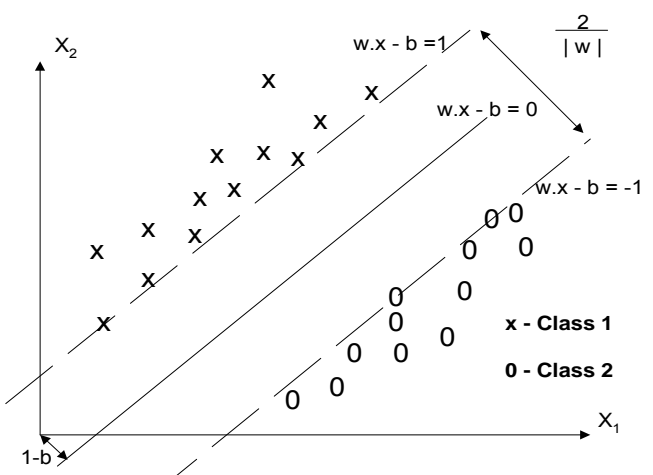

Figure 7. Maximum-margin hyperplane and margins for an SVM 
In this paper, the SVR predicts the existence of incipient faults in underground power cable systems by classifying the disturbances as either due to incipient or non-incipient faults. The SVR was developed based on the radial basis function (RBF) kernel as given in (16). The idea of the kernel function is to enable operations to be performed in the input space rather than the potentially high-dimensional feature space. The basic concept is to construct a mapping into a higher dimensional feature space by using reproducing kernels. However, the computation is still heavily dependent upon the number of training patterns, and generating a good data distribution for a high-dimensional problem will generally require a large training set. The bandwidth parameter, $\sigma$ and the regularization parameter, $\gamma$ associated with the RBF function are to be tuned in order to get the targeted results.

$$
k(x, y)=e^{-\left(\frac{|x-y|^{2}}{2 \sigma^{2}}\right)}
$$

In the SVR training, initially the bandwidth parameter, $\sigma$ and the regularization parameter, $\gamma$ was determined by trial and error and their values are found to be 0.2 and 10 respectively. For achieving the required SVR accuracy, the mean square error was chosen to be equal to 0.001 . Figure 8 shows the structure of SVR training and testing phases. The inputs to the SVR are feature values F1, F2, STMV and STFV obtained from the sample signals. While the output of SVR training are 0 or 1 indicating insipient fault and non insipient faults respectively from known events.

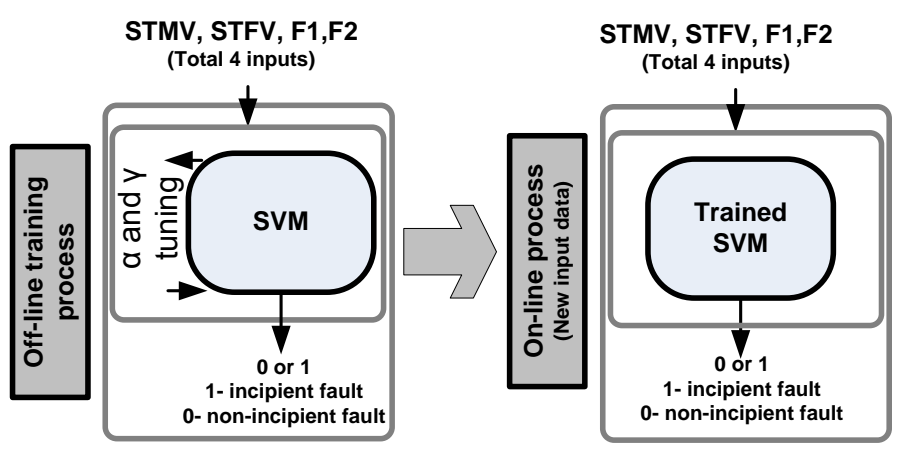

Figure 8. Training and testing process of SVR

\section{Test Results}

In this study, the outputs of the new prediction technique based on SVR are the existence of incipient and non-incipient faults. The performance of the SVR based ST is dependent on the training database developed based on the analyses performed on the 342 sets of voltage and current waveforms obtained from the power quality monitoring system of the Malaysian power utility. Table 4 shows the statistics for both the training and testing data for predicting the incipient faults. The testing data are the data to be predicted by the SVR. Here, the results of the ST for detecting incipient faults are first shown and then followed by the SVR results for predicting incipient faults.

Table 4. Number of training and testing data of SVR for predicting incipient faults

\begin{tabular}{|l|c|c|}
\hline \multicolumn{1}{|c|}{ Description } & $\begin{array}{c}\text { Number of training } \\
\text { data }\end{array}$ & $\begin{array}{c}\text { Number of } \\
\text { testing data }\end{array}$ \\
\hline $\begin{array}{l}\text { Non-incipient } \\
\text { fault }\end{array}$ & 255 & 219 \\
\hline Incipient fault & 87 & 50 \\
\hline Total & 342 & 269 \\
\hline
\end{tabular}




\section{A. Detection of Incipient Faults by the S-transform}

In Figure 9, the waveforms for both voltage and currents due to incipient fault are shown. This incipient fault was detected at the $11 \mathrm{kV}$ substation in Putrajaya, Malaysia. A high frequency current was detected in the blue phase and caused a minor voltage increase in the yellow phase voltage. The respective ST contours for the voltage and current waveforms are shown in Figure 10 and Figure 11, respectively. Both the ST contours were plotted for smaller windows of 0 to $500 \mathrm{~ms}$. In Figure 9, the ST contours show very short duration increases in the yellow phase voltage. The duration of the voltage increase was $12 \mathrm{~ms}$. The sudden voltage increase indicates the existence of high frequency current caused by a PD activity in the cable compartment because the frequency in per unit in this disturbance falls within range in Table 2 . In Figure 11, the existence of the high frequency current was also detected using the ST contours. The duration of the current was calculated to be $21 \mathrm{~ms}$.
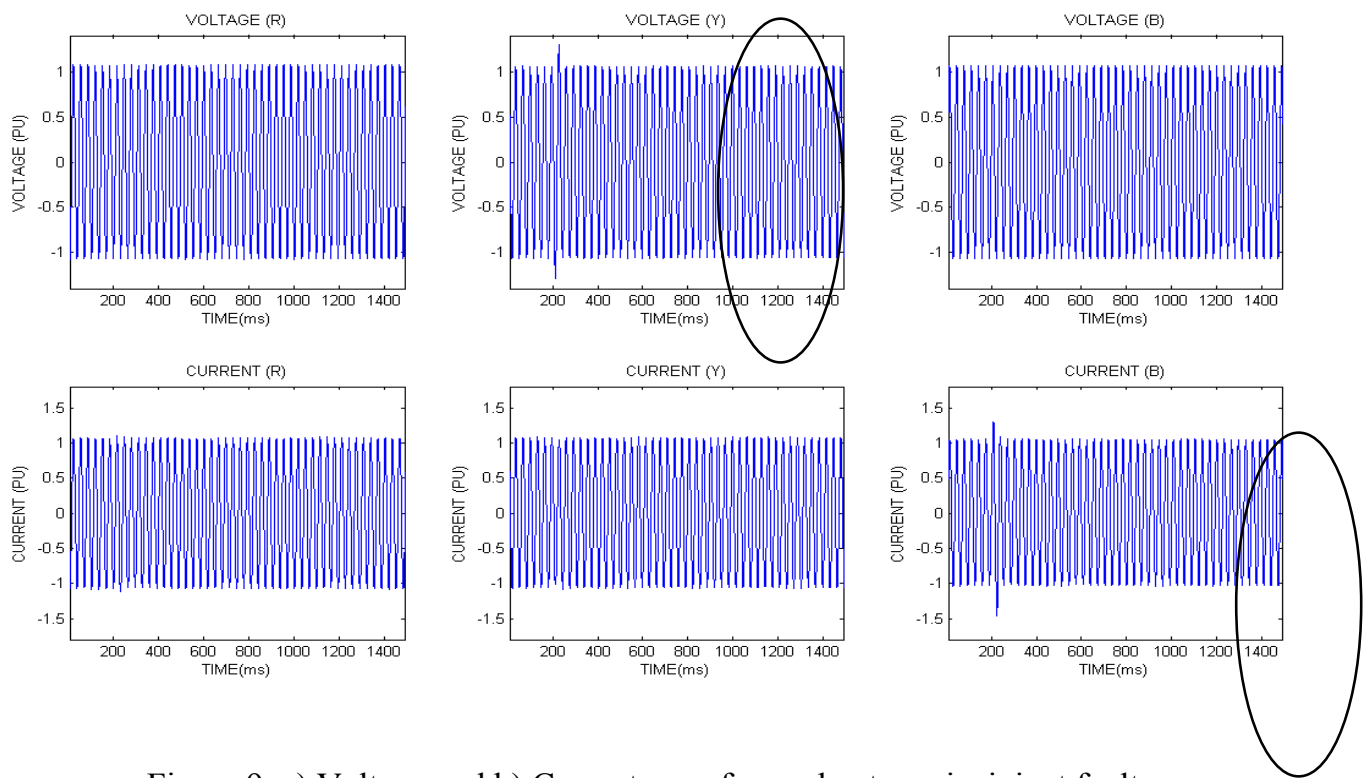

Figure 9. a) Voltage and b) Current waveforms due to an incipient fault
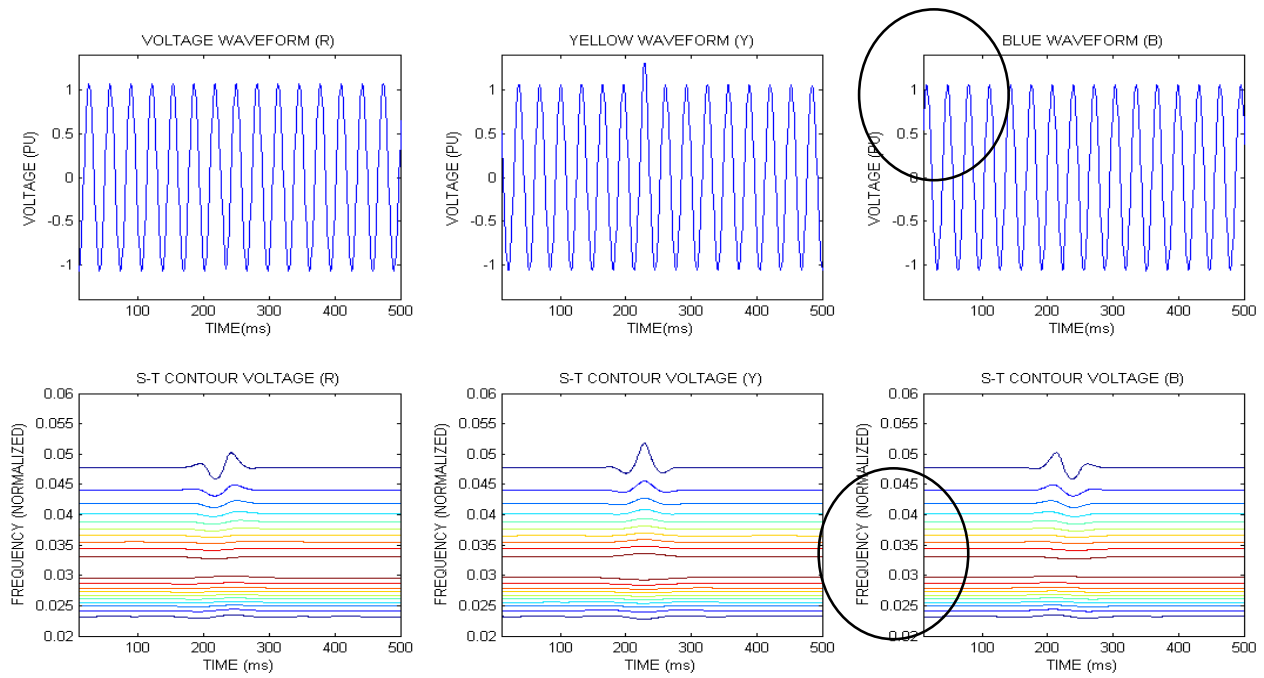

Figure 10. a) Voltage waveforms and b) ST contours for a 0-500 ms window 

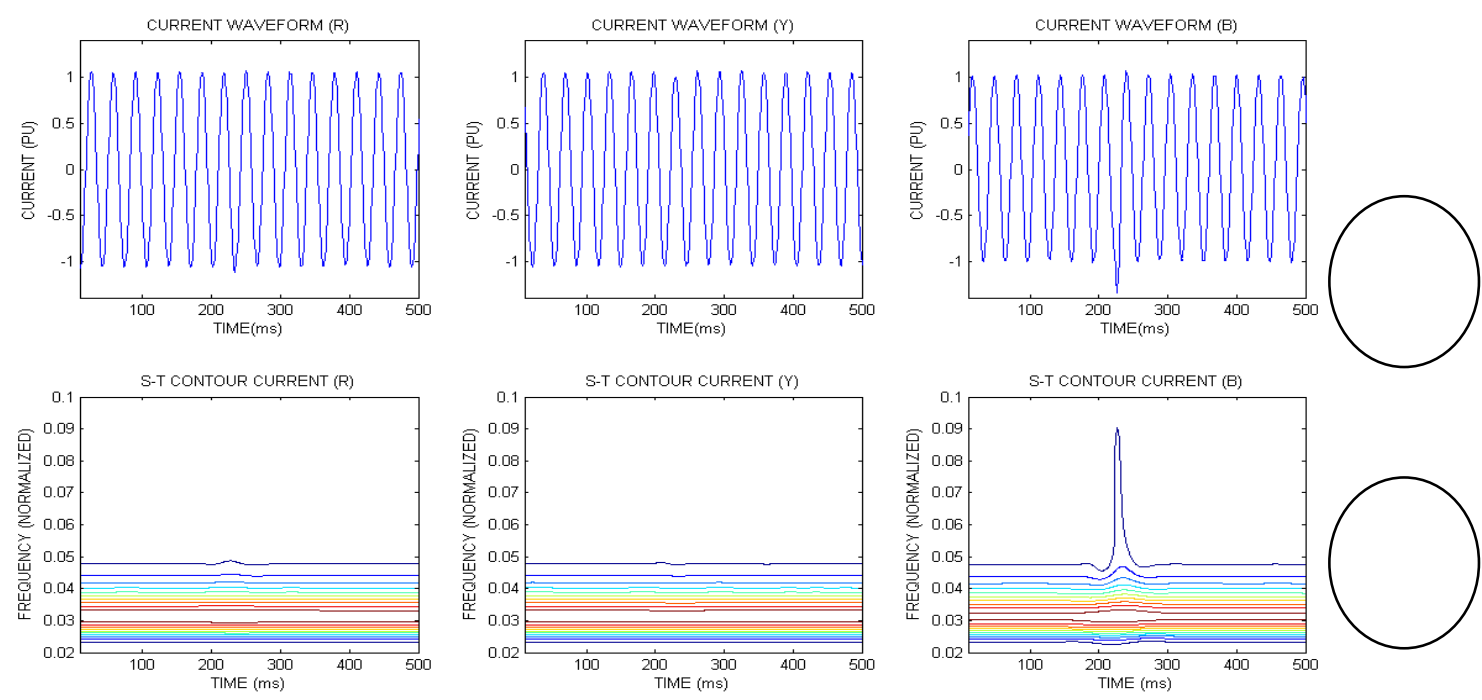

Figure 11. a) Current waveforms and b) ST contours for a 0-500 ms window

\section{B. SVR Results}

The effectiveness of the SVR in predicting the existence of incipient fault was tested with testing data collected from the power quality recorders. The results of testing the RBF SVR with 269 samples of PQ disturbance data with known causes as either due to non-incipient or incipient faults are shown in Table 5. The actual causes of the disturbances were verified based on the correlation done with the existing PQ database provided by the Malaysian power utility. From Table 5, the results of the SVR for predicting non-incipient and incipient faults give average accuracy of $94 \%$ and $86 \%$, respectively. There are 13 and 7 errors in predicting the non-incipient and incipient faults, respectively. The errors in predicting the non-incipient and incipient faults are probably due to the low feature values which are not within the specified range of feature values.

Table 5. Results of SVR for predicting incipient faults

\begin{tabular}{lcccc}
\hline Type of fault & $\begin{array}{c}\text { No. of } \\
\text { data }\end{array}$ & $\begin{array}{c}\text { Correct } \\
\text { prediction }\end{array}$ & $\begin{array}{c}\text { Wrong } \\
\text { prediction }\end{array}$ & $\begin{array}{c}\% \text { Accuracy in } \\
\text { prediction }\end{array}$ \\
\hline Non-incipient fault & 219 & 206 & 13 & $94 \%$ \\
Incipient fault & 50 & 43 & 7 & $86 \%$ \\
\hline
\end{tabular}

Based on these results, it is shown that the RBF SVR based ST is able to predict satisfactorily the existence of incipient faults. However, the accuracy of the SVR based ST results can be improved further by proper selection of ST features the kernel function and its parameters.

\section{Conclusion}

A novel approach to predict the existence of incipient fault was introduced by applying an advanced signal processing technique called the ST together with the SVR. The results of the studies performed in this research, showed that the new approach gives satisfactory accuracy in predicting incipient faults. The accuracy of the new approach is however dependent on the choice of kernel function, the parameters and the ST features selected. Based on these results, the RBF SVR based ST has the capability to perform real-time incipient fault diagnosis on large-scale power systems 


\section{References}

[1] BS EN 60270:2001/IEC 60270, High voltage test techniques - Partial discharge measurements, Section 3.1, 2000.

[2] Djokic, S.Z. \& Milanovic, J.V., "Advanced voltage sag characterization II: point of wave", IET Generation, Transmission and Distribution, Volume 1, No.1:146-153, 2007.

[3] Morel, O., Srinivas, N., Bernstein, B., "Partial Discharge Signals from TR-XLPE Insulated Cable", IEEE International Symposium on Electrical Insulation, Indianapolis, USA: 466 - 470, 2004.

[4] Weeks, W. L., Steiner, J. P., "Instrumentation for the detection and location of incipient faults on power cable", IEEE Trans. on Power Apparatus and Systems, Vol. PAS, 101, No.7:2328 -2335, 1982.

[5] Henriksen, M., Stone, G.C., Kurtz, M., "Propagation of Partial Discharge and Noise Pulses in Turbine Generators", IEEE Trans.on Energy Conversion, Vol. EC-1, No. 3:161-166, 1986.

[6] Kim, J.C., Lee, S.J., Kang, S. H., "Evaluation of feeder monitoring parameters for incipient fault detection using Laplace Trend Statistic", IEEE Trans. on Industry Applications, Vol.40, no.60:1-7, 2005.

[7] Dorris, D. L., Pace, M. O., Blalock, T.V., Alexeff, I., "Current pulses during water treeing detection system", IEEE Trans. on Dielectric and Electric Insulation, Vol.3, Issue 4: 515-522, 1996.

[8] Tanaka, T., "Space charge injected via interfaces and tree initiation in polymers", IEEE Trans. on Dielectric and Electric Insulation, Vol.8, Issue 5: 733-743, 2001.

[9] Ahmad, N., \& Srinivas, N., "On line Partial Discharge Detection in Cables", IEEE Conference on Electrical Insulation and Dielectric Phenomena, Minneapolis, USA: 214217, 1997.

[10] Srinivas, N., \& Bernstein, B.S., "Condition assessment of power cable system in energized state", 7th International Conference on Insulated Power Cable, Jicable'07, Versailles, France: 863-866, 2007.

[11] Denissov, D., Koehler, W., Hoek, S. M. M., Tenbohlen, S., Klein, T., "Online Partial Discharge Diagnostic for Cable Termination", XV International Symposium on High Voltage Engineering, University of Ljubljana, Elektroinštitut Milan Vidmar, Ljubljana, Slovenia:1-6, 2007.

[12] Kasztenny, B., Voloh, I., Jones, C. G., "Detection of Incipient Faults in Underground Medium Voltage Cables", Power Systems Conference, PSC '09:1 - 12, 2009.

[13] Butler, K.L., "An Expert System Based Framework for an Incipient Failure Detection and Predictive Maintenance System", Proc. Intelligent Systems Applications to Power Systems, ISAP '96: 321 - 326, 1996.

[14] Moghe, R., Mousavi, M. J., Stoupis, J., McGowan, J., "Field Investigation and Analysis of Incipient Faults Leading to a Catastrophic Failure in an Underground Distribution Feeder”, IEEE/PES Power Systems Conference and Exposition, PSCE '09:1 - 6, 2009.

[15] Gargoom, A. M., Eetugrul, N., Soong, W. L., "A Comparative study on effective signal processing tools", EPE 2005, Dresden, 2005.

[16] Faisal, M.F., Mohamed, A., "Comparing the performance of various mother wavelets in the detection of power quality disturbances in thre phase power systems", $2^{\text {nd }}$ IEEE International Power and Energy Conference, Johor Bahru, Malaysia, 1 - 3 Dec 2008.

[17] Faisal, M.F., Mohamed, A., "Identification of single and multiple power quality disturbances using S-transform and rule based classification", Journal of Applied Sciences 9(15): 2688-2700, 2009.

[18] Bhende, C. N., Mishra, S., Panigrahi, B.K., "Detection and classification of power quality disturbances using S-transform and modular neural network", Electric Power Systems Research, 78: 122-128, 2008.

[19] Stockwell, R.G., Mansinha, L., Lowe, R.P., "Localization of the Complex Spectrum: The S-transform”, IEEE Trans. on Signal Processing, Vol. 44, no.4: 998 - 1001, 1996. 
[20] Pinnegar, C.R, Mansinha, L., "The S-Transform with windows of arbitrary and varying shape", Geophysics vol.68: 381-385, 2003.

[21] Pillay, P., Bhattacharjee, A., "Application of Wavelet to Model Short Term Power System Disturbance", IEEE Transaction on Power System, vol.11, no.4, November 1996.

[22] Reddy, J. B., Mohanta, D. K., Karan, B. M., "Power System Disturbance Recognition Using Wavelet and S-transform Techniques", International Journal of Emerging Electric Power Systems 1(2): 1-6, 2004.

[23] Vapnik, V. Cortez, C., "Support-vector networks: Machine Learning", 20:273-297. http://citeseerx.ist.psu.edu/viewdoc/summary?doi=10.1.1.15.9362,1995.

[24] Drucker, H., Burges, C.J.C., Kaufman, L., Smola, A., \& Vapnik, V., "Support Vector Regression Machines", Available online: http://www.cs.cmu.edu/ pakyan/compbio/references/Drucker_NIPS_1996.pdf, 1998.

[25] Smola, A. \& Scholkopf, B., "A Tutorial on Support Vector Regression”, September 30, 2003, Statistics and Computing 14: 199-222, 2004, NeuroCOLT Technical Report TR98-030. ltext.pdf, 2003.

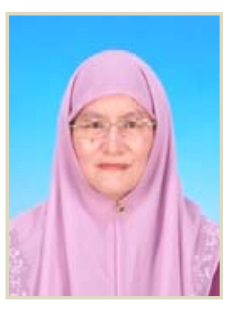

Azah Mohamed received her B.Sc from University of London in 1978 and M.Sc and Ph.D from Universiti Malaya in 1988 and 1995, respectively. She is a professor at the Department of Electrical, Electronic and System Engineering, Universiti Kebangsaan Malaysia. Her main research interests are in power system security, artificial intelligence, power quality and distributed generation. She is a senior member of IEEE.

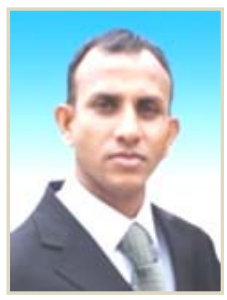

Hussain Shareef received his B.Sc with honours from IIT, Bangladesh, MS degree from METU, Turkey, and PhD degree from UTM, Malaysia, in 1999, 2002 and 2007, respectively. He is currently a lecturer in the Department of Electrical, Electronics, and Systems Engineering,Universiti Kebangsaan Malaysia. His current research interests are power system deregulation, power quality and power system distribution automation.

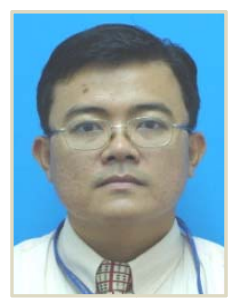

Mohamed Fuad Faisal received his BSc from CWRU in Cleveland, USA, M.Sc from UiTM Shah Alam Selangor and PhD from Universiti Kebangsaan Malaysia in 1991, 2002 and 2011, respectively. He is currently a Senior Manager Tenaga Nasional Bhd. 